\title{
A Revision of Preventive Web-based Psychotherapies in Subjects at Risk of Mental Disorders
}

\author{
Teresa Sánchez-Gutiérrez, $\mathrm{PhD}^{1,2}$; Sara Barbeito, $\mathrm{PhD}^{1}$; and Ana Calvo, $\mathrm{PhD}^{1}$ * \\ ${ }^{1}$ Facultad de Ciencias de la Salud. Universidad Internacional de la Rioja (UNIR) (Spain) \\ ${ }^{2}$ Department of Methodology and Behavioural Sciences. National Distance Education University \\ (UNED) (Spain)
}

Received 21 December 2016 | Accepted 1 November 2016 | Published 23 December 2016

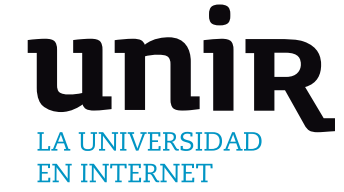

KEYWORDS

High-risk, Online

Psychotherapy,

Prevention, Web-based

Intervention.

OI: $10.9781 /$ ijimai.2017.459

\section{INTRODUCTION}

$\mathrm{P}$ SYCHOTHERAPEUTIC treatments are well established and have been recommended as the first option interventions, either independently or as pharmacological treatment coadyuvant, for the treatment of a wide range of mental disorders. This fact is included in recognized worldwide associations, protocols and guidelines such as APA [1] and NICE [36].

For the last years, the impulse of new technologies has overcome the traditional pathways of face-to-face clinical intervention and webbased psychological methodologies for intervention have started to gain success [31]. Neither are they a replacement of traditional psychotherapy, but indeed they has become a complementary intervention since online therapy could maximize the population scope [6]. Internet therapies can offer scalable approaches whereby large numbers of people can receive treatment and/or prevention, potentially bypassing barriers related to cost, location, lack of trained professionals, and stigma [3], [11]. However, despite the robust rationale and promising evidence, relatively few research has being conducted in healthy populations and in preventive health studies in individuals at risk of mental disorders.

This study aims to review the state-of-art about the effectiveness studies on preventive web-based interventions accomplished in samples of subjects at high risk for any mental disorder onset.

\section{METHOD}

A search was conducted in ProQuest, PubMed, Cochrane and

* Corresponding author.

E-mail address: anabelen.calvo@unir.net
Web of Science database for internet-based preventive psychological interventions that were published between 2010 and 2016.

Results showed that literature on this issue focuses in five domains: depressive symptoms, anxiety symptoms, eating behaviour disorder symptoms, problematic substance use and promotion of psychological well-being.

\section{EfFectiveness of Web-BASEd Psychotherapy in Individuals at Risk for Depression Symptoms}

Most of the reviewed studies on depression symptoms in high risk individuals showed positive effects of online interventions. ReyesPortillo et al. 2014 published a systematic review on 25 articles including web-based treatment and prevention programs for depression, anxiety, and suicide prevention in children, adolescents, and youth adults. They concluded that, even though depression and anxiety symptoms got reduced, there was limited evidence for the effectiveness of these kinds of interventions mainly due to the methodological design variations [40].

Within traditional therapies used for the treatment of depression disorders, Cognitive Behavioral Treatment (CBT) has proven to be the most effective and it is widely used by clinicians [8]. Internet-based Cognitive Behavioural Therapy (iCBT) has also been implemented for the decrease of depressive symptoms. Buntrock et al. 2016 performed a study comparing two groups of online intervention for the prevention of depression in 406 adults with subthreshold depression symptoms. Groups were randomized to either a web-based guided selfhelp intervention (cognitive-behavioral and problem-solving therapy supported by an online trainer) or a web-based psychoeducation program. They concluded that the use of a web-based guided self-help intervention compared with enhanced usual care reduced the incidence 
of MDD over 12 months. However, further research was needed to understand whether the effects are generalizable to both first onset of depression and depression recurrence as well as efficacy without the use of an online trainer [7]. Another study also found that the iCBT group had a significantly lower incidence of major depressive episodes at the 12-month follow-up than the control group in a study examining the effects of iCBT program on decreasing the risk of major depressive episodes among 20.000 workers employed in a private corporate group in [20]. Another study with 120 participants with mild to moderate depression were recruited from the general population and randomized to either guided ICBT or to live group treatment. Measures were completed before and after the intervention to assess depression, anxiety, and quality of life. Follow-ups were conducted at one-year and three-year after the treatment had ended. Results showed that both treatments were equally effective within the boundaries of non-inferiority and long-term effects could be sustained up to 3 years after treatment [2]. Moreover, Guille et at. 2015 designed a study to assess the effectiveness of a web-based cognitive behavioral therapy for the prevention of suicide in a sample of medical physicians at the beginning of their internships. Results suggested that this intervention helped reduce the he development of suicidal ideation among this population [18].

Third generation psychotherapies like acceptance and commitment therapy (ACT) are considered as empirically supported treatments with modest effect on managing depression [38]. Lappaplainen et al. 2015 investigated the effectiveness of an internet based ACT intervention in participants with depression symptoms. Conclusions of the study showed a decrease on depression symptoms which maintained through 12-month follow-up compared to the waitlist group [24].

Regarding non-functional depression symptoms, patients may experiment them as a consequence of organic disease. Thompson et al. (2015) implemented a study (Project UPLIFT) on the prevention of the onset of depression symptoms in patients with epilepsy. They performed a mindfulness-based cognitive therapy (MBCT) online intervention in a sample of 62 patients and concluded that the intervention reduced the number of symptoms of depression and increased patient's life satisfaction compared to the treatment as usual group [46].

Online interventions are also used in student populations who must cope with depression symptoms. A study analyzed whether an unguided Internet-based self-help intervention helped reduce symptoms of depression in a sample of at risk university students. Results showed that it was effective for the reduction of depression symptoms and negative thoughts [28]. Morgan et al. (2012) performed The Mood Memos study, a randomized controlled trial testing whether self-help behaviors for depression could be improved by promotional messages sent by email to 1326 participants. Authors concluded that these emails were found to reduce depression symptoms and psychological distress relative to control emails that provided information only [32].

Furthermore, current research conducted on this issue has already published protocols about future studies on the effectiveness of preventive internet-based psychotherapy in individuals at risk for depression [17], still with no evidence results.

\section{EfFectiveness of Web-Based Psychotherapy IN INDIVIDUALS AT RISK FOR ANXIETY DisORDERS}

Use of technology in psychotherapy has been a cost-effective way to disseminate empirically validated treatments for a wide variety of psychological problems, including anxiety disorders [35]. Christensen et al. 2014 tried to evaluate the effectiveness of a webbased program in preventing General Anxiety Disorder symptoms in a sample of young adults but they did not find significant differences in symptom reduction [10]. Another study was performed to establish the efficacy of two Internet-based prevention programs to reduce anxiety and depressive symptoms in adolescents. They concluded that both interventions reduced the depression and anxiety symptoms, but results should be cautiously interpreted because of the great loss-tofollow-up [49]. In the same line, in order to evaluate the efficacy of a transdiagnostic personality-trait-focused web-based intervention, a study was developed to reduce symptoms of common mental disorders, such as depression, anxiety, alcohol use and eating disorders in university students with a high risk for development. Results showed a reduction in depression and anxiety symptoms in the intervention group [34].

However, research in anxiety prevention has focused on the presence of symptoms or the high risk to develop posttraumatic stress disorders (PTSD). Kenardy et al. (2015) conducted a study on a web-based intervention to prevent long-term children PTSD. They concluded that intervention diminished the occurrence of trauma symptoms. Furthermore, when initial distress was not elevated, no significant differences were noted between the intervention and control group. Therefore, these results indicate the efficacy of early online intervention in children presenting with the specific risk factor of high initial distress [21]. However, other study tried to determine whether an internet based intervention called Trauma TIPS was effective in preventing the onset of PTSD symptoms in injury patients. Results showed that the program was not significantly effective in the reduction of PTSD onset and proposed that future research should add gameplay, a blended care context, and targeting high-risk individuals who would be more likely to benefit from the intervention [33]. In the same line, Marsac et al. 2013 conducted a study which aimed to evaluate the efficacy of a web-based psychoeducational intervention for parents in promoting emotional recovery following pediatric injury. They found ambiguous results and concluded that this intervention was insufficient in the prevention of PTSD symptoms [30]. Moreover, a protocol of a study about the prevention of PTSD in individuals who had a level 1 traffic accident was developed [16]. Results have not been published yet.

\section{Effectiveness of Web-Based Psychotherapy in Individuals at Risk for Fresenting Symptoms of EATING DISORDER}

Body image has become an important and overvalued issue in the general population. It is usual that people present concerns about body image but, in some cases, this worry could become pathological. A distortional body image is frequently present in eating disorders and in body dysmorphic disorder. Intervention in people at risk for the presence of symptoms of these disorders is crucial to minimize their impact on well-being. A study compared two groups (internet-based cognitive therapy and waitlist group) with binge eating disorder. The main findings showed that the online intervention significantly reduced the number of objective binge-eating episodes over the last 28 days in the treatment groups compared with the waitlist group. Reductions in the number of binge-eating episodes could be maintained from posttreatment to 12-month follow-up. Furthermore, the intervention significantly reduced scores on all secondary eating psychopathology outcomes [48].

Moreover, internet-based interventions on obesity could be effective and positively influence the decrease of associated stress symptoms, mainly related to the problems derived from a disturbed body image. Lappalainen et al. 2014 published a study protocol to test whether different interventions (including a web-guided Cognitive Behavioral Therapy-based (CBT) intervention) were effective in the promotion of physical and psychological well-being of a sample of obese and stressed individuals. Outcomes from the study are being developed [25]. 
VI. Effectiveness of Web-Based Psychotherapy in Individuals at Risk for Substance Use Disorders

One of the most developed body of research on the effectiveness of online psychotherapy is the one related to substance use, mainly alcohol misuse. A systematic review on mobile technology-based interventions for adult alcohol users showed positive results on alcohol use reduction after interventions. However, because of methodological issues, these are preliminary results that should widely be investigated in future studies [15]. Another study aimed to evaluate the effectiveness of a computer-based intervention for the reduction of alcohol use in a sample of adolescents. Conclusions suggested that it could be a suitable orientation tool used to reduce the alcohol intake and binge drinking in adolescents [13].

Arnaud et al. (2016) evaluated the effectiveness of a 3-month follow-up fully automatized web-based brief intervention based on the motivational interviewing for a sample of 1449, 16-18year-old substance-using adolescents compared to controls with only assessment measures. They found a reduction in alcohol drinking and less existing substance use service barriers for at-risk drinking adolescents [4]. On the contrary, another study found no strong evidence of effectiveness of their study (AMADEUS-2) where they implemented an online alcohol intervention among studies with risky drinking behavior [5]. In the same line, Kypri et al. (2014) designed a web-based alcohol screening and psychoeducational brief intervention on the risky alcohol drinking in 3422 university students who were followed up to six months. They found a small reduction in the amount of alcohol consumed but not in other alcohol consumption and problem measures [22].

Likewise, current protocols designed to analyze the effectiveness of an internet-based self-help intervention to reduce co-occurring alcohol misuse and depression symptoms in adults in comparison to a waitlist are still in progress [41].

Regarding the online prevention of tobacco use onset, studies are scarce. Cremers et al. 2015 found that a web-based prevention program for adolescents at risk of tobacco initiation was not effective in the reduction of children's intention to smoke and smoking behavior after 12 and 25 months of follow-up compared to the no information group [9]. Moreover, Lana et al. 2013 conducted a study to assess the impact of a web-based intervention supplemented with text messages to reduce cancer risk linked with smoking, unhealthy diet, alcohol consumption, obesity, sedentary lifestyle and sun exposure. They concluded that the web-based intervention supplemented with text messages had a positive global impact, but it leads to only minimal changes in risky behaviors. This intervention appears useful in controlling overweight adolescents [23].

Other drugs internet-based prevention treatment has not been fully investigated yet. An online intervention (Can Reduce) with self-help intervention was achieved to reduce cannabis use comparing the inclusion or absence of chat counselling in a group of risky heavycannabis users. Conclusions suggested a reduction of the amount of cannabis use in those participants who also used the online chat [43]. This team also is currently analyzing the same online intervention in participants with cocaine use. They aim to investigate the effectiveness of the web-based self-help intervention, Snow Control 2.0 with tailored chat counseling, which is based on CBT, Motivational Enhancement Therapy (MET), Behavioral Self-Management (BSM), and social problem solving. The objective of this protocol is to observe a reduction in the amount of cocaine use in at risk patients. Results of this research are still in progress [42]

Concerning polyconsumption, Schwinn et al. (2015) focused their attention in the prevention of drug use among sexual-minority youths, who were at risk of developing high substance abuse. They applied a web-based 3-session prevention program and concluded that at 3-month follow-up, adolescents reported less stress and less peer drug use, as well as higher coping, problem solving, and drug-use refusal skills. Efficacy of this program has not been analyzed yet [45]. This team also conducted a study on internet-based drug prevention among adolescent girls. They observed that girls from the intervention group reported lower rates of substance use and achieved gains over girls in the control group on normative beliefs and self-efficacy at posttest and 6-month follow-up, respectively [44].

\section{Web-based Psychological Health Promotion in the General Population}

Internet-based intervention not only provides new delivery media for mental health treatments, it also opens the possibility for developing health promotion in the general population. The effects of an online positive psychology program (Bite Back) for the improvement of the well-being and mental health in a sample of Australian youth have been examined. Conclusions showed that this program decreased symptoms of depression and stress and increased well-being in young people, especially for those who used the website for 30 minutes [29].

University students' health promotion has been another focus of interest. One study was conducted to evaluate the efficacy of an Acceptance and Commitment Therapy (ACT) web-based program (The Student Compass) in order to increase university students' wellbeing. Results revealed a reduction in symptoms of stress, anxiety and depression within the intervention group [39]. Another study with the same aim found a decrease on the frequency of depression and anxiety symptoms in the ACT group relative to the waitlist group [27].

\section{Computational Techniques for the AnAlysis of} Web-BAsed Psychological InTERVENTIONS

The online interventions previously described generate a secondary (but not for this reason useless) extremely large amount of data regarding different aspects of the people under online treatment, from personal preferences used for marketing purposes to the confirmation of the presence of symptoms or diseases that could be altogether glued as jigsaw pieces[50].

The term "Big data" is becoming frequent in the psychology field, as it could be useful for the selection of effective web-based psychotherapies that had proven to be effective in population samples analyzed by data mining tools. The use of data mining in psychotherapy is still scarce, although some studies have found interesting usages for the health promotion. As an example, in a study conducted with data collected from elderly people under self-care programs which was analyzed with web usage mining, results showed association relationships, interest and sequence-based representation schemes that could be used for research in medicine, public health, nursing and psychology and for policy-making in the health care domain[19].

Another application of big data deals with the designing of instruments for the assessment of knowledge and behaviors. Preventive web-based intervention assessment tools could develop from studies whose main objective was to elaborate algorithms for the optimal selection of available items in questionnaires related to psychology and education subjects [12][37].

\section{DiscusSION}

Web-based psychological interventions for the prevention of mental disorders seem to be effective for at risk individuals. Online health promotion in the general population is also effective to avoid the onset of clinical psychological circumstances. Moreover, internet- 
based psychological intervention works as a suitable way for the economization and optimization of results. It could reach a variety of population: from those who present difficulties for the access of health promotion and prevention (those with mobility troubles and geographical barriers, lack of economic resources, stigma, special personality traits...), symptomatic patients, but also at risk individuals or people just interested in the improvement of their quality of life. This psychotherapy format could work for non-clinic intervention, such as counselling or coaching [43].

Despite the abundant research that just recently started on this topic, more body of research is needed in order to unify and structure methodology designs and increase the number of participants. On this regarding, a deep understanding of the user needs and preferences is needed to increase the participation and adherence of people to internet interventions, together with an actively involving of users in designing processes from the outset [14]. Technologies such as data mining techniques would help maximize the processing of large volumes of research data and would result in more accurate population representations and worldwide conclusions of web-based psychotherapy studies [26].

Promising directions of research include a greater focus on usercentered approaches (including user-centered design, intervention individualization, and design of modularized programs), increased emphasis on engagement (including methods such as gaming, telepresence and persuasive technology, and measuring engagement), increased international and intersectorial collaboration, and rapid testing and implementation [14].

AcKnowledgment

This work is partially funded by Universidad Internacional de La Rioja, UNIR (UNIR, http://www.unir.net), under the Research Support Strategy: Emergent Research Group (2016-2018).

\section{REFERENCES}

[1] A. P. A. Presidential Task Force on Evidence-Based Practice, Evidencebased practice in psychology, Am Psychol, vol 61, no 4, 271-285, 2006.

[2] G. Andersson, H. Hesser, A. Veilord, L. Svedling, F. Andersson, O. Sleman, et al., Randomised controlled non-inferiority trial with 3-year follow-up of internet-delivered versus face-to-face group cognitive behavioural therapy for depression, J Affect Disord, vol 151, no 3, pp 986-994, 2013.

[3] L.H. Andrade, J. Alonso, Z. Mneimneh, J.E. Wells, A. Al-Hamzawi, G. Borges, et al., Barriers to mental health treatment: results from the WHO World Mental Health surveys, Psychol Med, vol 44, no 6, pp 1303-1317, 2014.

[4] N. Arnaud, C. Baldus, T.H. Elgan, N. De Paepe, H. Tonnesen, L. Csemy, L., et al., Effectiveness of a Web-Based Screening and Fully Automated Brief Motivational Intervention for Adolescent Substance Use: A Randomized Controlled Trial, J Med Internet Res, vol 18, no 5, e103, 2016.

[5] P. Bendtsen, M. Bendtsen, N. Karlsson, I.R. White and J. McCambridge, Online Alcohol Assessment and Feedback for Hazardous and Harmful Drinkers: Findings from the AMADEUS-2 Randomized Controlled Trial of Routine Practice in Swedish Universities, J Med Internet Res, vol 17, no 7, e170, 2015.

[6] E. Bunge, P. López, J. Mandil, M. Gomar, R. Borgialli, Actitudes de los terapeutas argentinos hacia la incorporación de nuevas tecnologías en psicoterapia, Revista Argentina de Clínica Psicológica, vol 18, no 3, pp 209-216, 2009.

[7] C. Buntrock, D.D. Ebert, D. Lehr, F. Smit, H. Riper, M. Berking and P. Cuijpers, Effect of a Web-Based Guided Self-help Intervention for Prevention of Major Depression in Adults with Subthreshold Depression: A Randomized Clinical Trial. JAMA, vol 315, no 17, pp 1854-1863, 2016.

[8] A.C. Butler, J. E. Chapman, E.M. Forman and A. T. Beck, The empirical status of cognitive-behavioral therapy: a review of meta-analyses. Clin Psychol Rev, vol 26, no 1, pp 17-31, 2006.
[9] H.P. Cremers, L. Mercken, M. Candel, H. de Vries and A. Oenema, A Web-based, computer-tailored smoking prevention program to prevent children from starting to smoke after transferring to secondary school: randomized controlled trial. J Med Internet Res, vol 17, no 3, e59, 2015.

[10] H. Christensen, P. Batterham, A. Mackinnon, k.M. Griffiths, K.J. Kalia Hehir, J. Kenardy, et al., Prevention of generalized anxiety disorder using a web intervention, iChill: randomized controlled trial. J Med Internet Res, vol 16, no 9, e199, 2014.

[11] H. Christensen and I.B. Hickie, E-mental health: a new era in delivery of mental health services, Med J Aust, vol 192, no 11 Suppl, S2-3, 2010.

[12] V. Crisp, C. Ward, The development of a formative scenario- based computer assisted assessment tool in psychopatology for teachers: The PeCPAA project. Computers and education, vol 50, 1509-1526, 2008.

[13] R. M. Drost, A.T. Paulus, A. F., Jander, L. Mercken, H. de Vries, D. Ruwaard and S. M. Evers. A Web-Based Computer-Tailored Alcohol Prevention Program for Adolescents: Cost-Effectiveness and Intersectoral Costs and Benefits, J Med Internet Res, vol 18, no 4, e93, 2016.

[14] T. M. Fleming, D. de Beurs, Y. Khazaal, A. Gaggioli, G. Riva, C. Botella et al., Maximizing the Impact of e-Therapy and Serious Gaming: Time for a Paradigm Shift, Front Psychiatry,vol 7, 65, 2016.

[15] L. A. Fowler, S.L. Holt, D. Joshi, Mobile technology-based interventions for adult users of alcohol: A systematic review of the literature. Addict Behav,vol 62, pp 25-34, 2016.

[16] S. A. Freedman, E. Dayan, Y.B. Kimelman, H. Weissman and R. Eitan, Early intervention for preventing posttraumatic stress disorder: an Internetbased virtual reality treatment. Eur J Psychotraumatol, vol 6, 2015.

[17] T.G. Gladstone, M. Marko-Holguin, P. Rothberg, J. Nidetz, A. Diehl, D. T. DeFrino, D. T. et al., An internet-based adolescent depression preventive intervention: study protocol for a randomized control trial. Trials, vol 16, pp 203, 2015.

[18] C. Guille, Z. Zhao, J. Krystal, B. Nichols, K. Brady and S. Sen, WebBased Cognitive Behavioral Therapy Intervention for the Prevention of Suicidal Ideation in Medical Interns: A Randomized Clinical Trial, JAMA Psychiatry,vol 72, no 12,pp 1192-1198, 2015.

[19] Y-S, Hung, B. Kuei-Ling, Chen, C-T, Yang, G-F Deng, Web usage mining for analysing elder self-care behavior patterns. Reference Expert Systems with Applications, vol 40, 775-783, 2013.

[20] K. Imamura, N. Kawakami, T. A. Furukawa,Y. Matsuyama, A. Shimazu, R. Umanodan, et al., Does Internet-based cognitive behavioral therapy (iCBT) prevent major depressive episode for workers? A 12-month follow-up of a randomized controlled trial, Psychol Med, vol 45, no 9, pp 1907-1917, 2015.

[21] J. A. Kenardy, C. M. Cox and F.L. Brown, A Web-Based Early Intervention Can Prevent Long-Term PTS Reactions in Children with High Initial Distress Following Accidental Injury., J Trauma Stress, vol 28, no 4, pp 366-369, 2015.

[22] K. Kypri, T. Vater, S. J. Bowe, J. B. Saunders, J. A., Cunningham, N. J. Horton and J. McCambridge, Web-based alcohol screening and brief intervention for university students: a randomized trial, JAMA, vol 311, no 12, pp 1218-1224, 2014.

[23] A. Lana, G. Faya-Ornia and M. L. Lopez, Impact of a web-based intervention supplemented with text messages to improve cancer prevention behaviors among adolescents: results from a randomized controlled trial, Prev Med, vol 59, pp 54-59, 2014.

[24] P. Lappalainen, S. Langrial, H. Oinas-Kukkonen, A. Tolvanen and R. Lappalainen, Web-based acceptance and commitment therapy for depressive symptoms with minimal support: a randomized controlled trial, Behav Modif, vol 39, no 6, pp 805-834, 2015.

[25] R. Lappalainen, E. Sairanen, E. Jarvela, S. Rantala, R. Korpela, S. Puttonen et al., The effectiveness and applicability of different lifestyle interventions for enhancing wellbeing: the study design for a randomized controlled trial for persons with metabolic syndrome risk factors and psychological distress, BMC Public Health, vol 14, pp 310, 2014.

[26] S-H. Liao, P-H. Chu, P-Y. Hsiao, Data mining techniques and applications - A decade review from 2000 to 2011. Expert Systems with Applications, vol 39,11303-11311, 2012.

[27] M. E. Levin, J. Pistorello, J. R. Seeley and S.C. Hayes, Feasibility of a prototype web-based acceptance and commitment therapy prevention program for college students. J Am Coll Health,vol 62, no 1,pp 20-30, 2014.

[28] O. K. Lintvedt, K.M. Griffiths, K. Sorensen, A. R. Ostvik, C.E. Wang, 
M. Eisemann, and K. Waterloo, Evaluating the effectiveness and efficacy of unguided internet-based self-help intervention for the prevention of depression: a randomized controlled trial, Clin Psychol Psychother, vol 20, no 1, pp 10-27, 2013.

[29] V. Manicavasagar, D. Horswood, R. Burckhardt, A. Lum, D. HadziPavlovic and G. Parker, Feasibility and effectiveness of a web-based positive psychology program for youth mental health: randomized controlled trial, J Med Internet Res, vol 16, no 6, e140, 2014.

[30] M. L. Marsac, A. K. Hildenbrand, K. L. Kohser, F. K., Winston, Y. Li and N. Kassam-Adams, Preventing posttraumatic stress following pediatric injury: a randomized controlled trial of a web-based psycho-educational intervention for parents. J Pediatr Psychol, vol 38, no 10, pp 1101-1111, 2013.

[31] D. C. Mohr, M.N. Burns, S. M. Schueller, G. Clarke and M. Klinkman, Behavioral intervention technologies: evidence review and recommendations for future research in mental health. Gen Hosp Psychiatry, vol 35, no 4, pp 332-338, 2013.

[32] A. J. Morgan, A. F, Jorm and A. J. Mackinnon, Email-based promotion of self-help for subthreshold depression: Mood Memos randomised controlled trial, Br J Psychiatry, vol 200, no 5, pp 412-418, 2012.

[33] J. Mouthaan, M. Sijbrandij, G. J. de Vries, J. B. Reitsma, R. van de Schoot, J. Goslings, et al., Internet-based early intervention to prevent posttraumatic stress disorder in injury patients: randomized controlled trial. J Med Internet Res, vol 15, no 8, e165, 2013.

[34] P. Musiat, P. Conrod, J. Treasure, A. Tylee, C. Williams, U, Schmidt, Targeted prevention of common mental health disorders in university students: randomised controlled trial of a transdiagnostic trait-focused web-based intervention. PLoS One, vol 9, no 4, e93621, 2014.

[35] M. G. Newman, L.E. Szkodny, S. J. Llera and A. Przeworski, A review of technology-assisted self-help and minimal contact therapies for anxiety and depression: is human contact necessary for therapeutic efficacy? Clin Psychol Rev, vol 31, no 1, pp 89-103, 2011.

[36] NICE guidelines, Common mental health disorders: identification and pathways to care, NICE guidelines [CG123], 2011.

[37] M. Pérez, Las Terapias de tercera generación como terapias contextuales. Madrid: Sintesis, 2014.

[38] J. Pereira, M. Vilâ, Variable neighborhood search heuristics for a test assembly design problema. Expert Systems with Applications vol 42, 4805-4817, 2015.

[39] P. Rasanen, P. Lappalainen, J. Muotka, A. Tolvanen and R. Lappalainen, $\mathrm{R}$, An online guided ACT intervention for enhancing the psychological wellbeing of university students: A randomized controlled clinical trial, Behav Res Ther,vol 78, pp 30-42, 2016.

[40] J. A. Reyes-Portillo, L. Mufson, L.L. Greenhill, M. S. Gould, P. W. Fisher, N. Tarlow, N and M.A. Rynn, Web-based interventions for youth internalizing problems: a systematic review, J Am Acad Child Adolesc Psychiatry, vol 53, no 12, pp 1254-1270 e1255, 2014.

[41] M. P. Schaub, M. Blankers, D. Lehr, L. Boss, H. Riper, J. Dekker, J., et al., Efficacy of an internet-based self-help intervention to reduce co-occurring alcohol misuse and depression symptoms in adults: study protocol of a three-arm randomised controlled trial, BMJ Open, vol 6, no 5, e011457, 2016.

[42] M. P. Schaub, L. J. Maier, A. Wenger, L. Stark, O. Berg, T. Beck, et al., Evaluating the efficacy of a web-based self-help intervention with and without chat counseling in reducing the cocaine use of problematic cocaine users: the study protocol of a pragmatic three-arm randomized controlled trial, BMC Psychiatry, vol 15, pp 156, 2015.

[43] M. P. Schaub, A. Wenger, O. Berg, T. Beck, L. Stark, E. Buehler and S. Haug, A Web-Based Self-Help Intervention with and Without Chat Counseling to Reduce Cannabis Use in Problematic Cannabis Users: Three-Arm Randomized Controlled Trial, J Med Internet Res, vol 17, no 10, e232, 2015.

[44] T. M. Schwinn, J. E. Hopkins and S. P. Schinke, Developing a Web-Based Intervention to Prevent Drug Use among Adolescent Girls, Res Soc Work Pract, vol 26, no 1, pp 8-13, 2016.

[45] T.M. Schwinn, B. Thom, S. P. Schinke and J. Hopkins, Preventing drug use among sexual-minority youths: findings from a tailored, web-based intervention, J Adolesc Health,vol 56, no 5,pp 571-573, 2015.

[46] N. J. Thompson, A. H. Patel, L. M. Selwa, S. C. Stoll, C.E. Begley, E. K. Johnson and R. T. Fraser, Expanding the efficacy of Project UPLIFT: Distance delivery of mindfulness-based depression prevention to people with epilepsy, J Consult Clin Psychol, vol 83, no 2, pp 304-313, 2015.

[47] A. Trujillo, Nuevas tecnologías y Psicología. Una perspectiva actual, Apuntes de Psicología, vol 23, no 3, pp 321-335, 2005.

[48] B. Wagner, M. Nagl, R. Dolemeyer, G. Klinitzke, J. Steining, A. Hilbert, A. Kersting, Randomized Controlled Trial of an Internet-Based CognitiveBehavioral Treatment Program for Binge-Eating Disorder, Behavior Therapy, vol 47, pp 500-514, 2016.

[49] N. Wong, L.Kady, L. Mewton, M. Sunderland, G. Andrews, Preventing Anxiety and Depression in Adolescents: A Randomised Controlled Trial of Two School Based Internet-Delivered Cognitive Behavioural Therapy Programmes, Internet Interventions, vol 1, pp 90-94, 2014.

[50] I.Yaqooba, I. Abaker Targio Hashema, A. Gania, S. Mokhtara,Ejaz Ahmeda, N. Badrul Anuara, A. V. Vasilakosb, Big data: From beginning to futureInternational Journal of Information Management, vol 36,1231$1247,2016$.

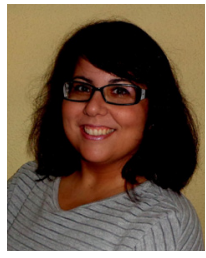

\section{Teresa Sanchez-Gutiérrez}

Teresa Sanchez-Gutiérrez was born in 1984. She received her $\mathrm{PhD}$ from the Complutense University in Madrid (Psychopharmacology and substance abuse) in 2014. She worked as a researcher and psychotherapist at the Child and Adolescent Psychiatry Department, Hospital General Universitario Gregorio Marañón from 2010 to 2015. Since 2015, she became Professor and tutor at the National Distance Education University (UNED) in Córdoba and since 2016 she is Professor at Universidad Internacional de la Rioja (UNIR). She has contributed to scientific research with several manuscripts, book chapters and lectures at international and national congresses.

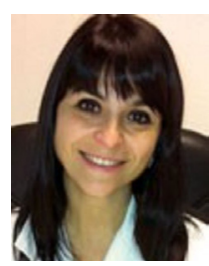

\section{Sara Barbeito}

Sara Barbeito was born in 1981. She received her $\mathrm{PhD}$ from the Medicine University of Bask country (neuroscience) in 2014. From 2004 to 2015 she was a researcher in the Santiago Apostol Hospital of Vitoria (CIBERSAM 10 group). Since 2015 she is Professor in the Rioja International University (UNIR). She has numerous international articles and book's chapters.

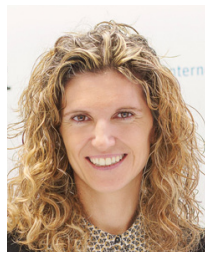

Ana Calvo

Ana Calvo PhD is full Professor and Director of The Master's in General Health Psychology at Universidad Internacional de la Rioja (UNIR) since 2015. She received her $\mathrm{PhD}$ from the Complutense University in Madrid in 2013. She worked as a researcher and psychotherapist at the Child and Adolescent Psychiatry Department, Hospital General Universitario Gregorio Marañón from 2009 to 2016. In addition, she was working during her international predoctoral fellowship in different early psychosis services for young people at Orygen Youth Health Clinical and research Centre and Melbourne University in Melbourne, Australia. (2012-2013) and She was working as a Post-doctoral Researcher fellow at Royal College of Surgeons in Ireland and Trinity College of Dublin in Dublin, Ireland (2015). She has contributed to scientific research with several manuscripts, book chapters and lectures at international and national congresses. 